\title{
The Reaction of Cyanoacetylhdrazine with Furan-2-Aldehyde: Novel Synthesis of Thiophene, Azole, Azine and Coumarin Derivatives and Their Antitumor Evaluation
}

\author{
Wagnat W. Wardakhan ${ }^{1}$, Sherif M. Sherif ${ }^{2}$, Rafat M. Mohareb ${ }^{2^{*}}$, Amr S. Abouzied ${ }^{1}$ \\ ${ }^{1}$ National Organization for Drug Control \& Research, Cairo, Egypt \\ ${ }^{2}$ Department of Chemistry, Faculty of Science, Cairo University, Giza, Egypt \\ Email: *raafat_mohareb@yahoo.com
}

Received August 20, 2012; revised September 27, 2012; accepted October 16, 2012

\begin{abstract}
The reaction of cyanoacetylhydrazine $\mathbf{1}$ with furan-2-aldehyde $\mathbf{2}$ gives the hydrazide-hydrazone derivative $\mathbf{3}$. The latter compound undergoes a series of heterocyclization reactions to give new heterocyclic compounds. The antitumor evaluation of the newly synthesized products against three cancer cell lines, namely breast adenocarcinoma (MCF-7), nonsmall cell lung cancer (NCI-H460) and CNS cancer (SF-268) were recorded. Some of the synthesized compounds show high inhibitory effects.
\end{abstract}

Keywords: Acetylhydrazine; Hydrazide-Hydrazone; Thiophene; Thiazole; Pyridine; Coumarin; Antitumor

\section{Introduction}

Substituted aminothioxomethyl hydrazides are important building blocks in synthetic heterocyclic chemistry. The $\mathrm{S} / \mathrm{N}$ regioselective nucleophilic competition in the synthesis of heterocyclic systems by intermolecular and intramolecular cyclization, as well as the change in reaction conditions which might favor N-attack or S-attack or even attack on the substituted terminals are important factors for the diversity of the produced heterocyclic systems from the title reaction precursor. On the other hand, the synthetic potential and biological activity of several heterocyclic related to the named hydrazides have been explored to the maximum extent. Among the pharmacological profiles are their antimicrobial [1,2], antitubercular $[3,4]$, anticonvulsant $[5,6]$ anti-inflammatory $[7$, 8], antidepressant [9], antitumor [10], and analgesic activities [11]. Continuing our interest in developing new heterocyclic systems based on hydrazide-hydrazones [12, 13] as well as design and synthesis of heterocyclic with promising biological activities [14-17], we focused our work on developing novel polyfunctionalized heterocyclic compounds with potential bioactivity.

\section{Results and Discussion}

In this work we report the reaction of cyanoacetylhydra-

"Corresponding author. zine (1) with furan-2-aldehyde (2) to give the hydrazidehydrazone derivative 3 , the structure of which was confirmed on the basis of analytical and spectral data. Thus, the ${ }^{1} \mathrm{H}$ NMR spectrum showed a singlet at $\delta 6.60(2 \mathrm{H})$ ppm corresponding to $\mathrm{CH}_{2}$ group, a multiplet at $\delta 6.52$ $7.88(3 \mathrm{H}) \mathrm{ppm}$ corresponding to furan protons, a singlet at $\delta 7.54(1 \mathrm{H}) \mathrm{ppm}$ corresponding to $\mathrm{CH}$ group, and a singlet $\left(\mathrm{D}_{2} \mathrm{O}\right.$-exchangeable $)$ at $\delta 11.62(1 \mathrm{H}) \mathrm{ppm}$ corresponding to $\mathrm{NH}$ group.

The formed hydrazide-hydrazone derivative $\mathbf{3}$ was subjected through a series of heterocyclization reactions to form products with expected pharmaceutical applications. Thus, condensation of $\mathbf{3}$ with benzaldehyde (4) in ethanolic/piperidene solution gave the benzylidene derivative $\mathbf{5}$. The reaction of compound $\mathbf{5}$ with either hydrazine hydrate (6a) or phenylhydrazine (6b) gave the pyrazole derivatives $7 \mathbf{a}$ and $\mathbf{7 b}$, respectively.

On the other hand, the reaction of compound 5 with either malononitrile (8a) or ethyl cyanoacetate (8b) gave the pyridone derivatives 10a and 10b, respectively. The reaction took place via 1,6-intramolecular dipolar cyclization of the intermediate 9a,b (cf. Scheme 1), analyticcal and spectral data of the latter compounds were consistent with the assigned structures. Thus, the ${ }^{1} \mathrm{H}$ NMR spectrum of 10b showed, a triplet at $\delta 1.29(3 \mathrm{H}) \mathrm{ppm}$ indicating the presence of the $\mathrm{CH}_{3}$ group, a quartet at $\delta$ $4.24(2 \mathrm{H}) \mathrm{ppm}$ corresponding to $\mathrm{CH}_{2}$ group, a multiplet at $\delta 6.52-7.88(3 \mathrm{H}) \mathrm{ppm}$ for furan protons, a singlet 
<smiles>N#CCC(=O)NN</smiles>

1<smiles>O=Cc1ccco1</smiles>

2<smiles>N#CCC(=O)N/N=C/c1ccco1</smiles>

3<smiles>[R]NN=C=NNC(=O)c1c(N)nn([R])c1-c1ccc(C=NN=Cc2ccco2)cc1</smiles>

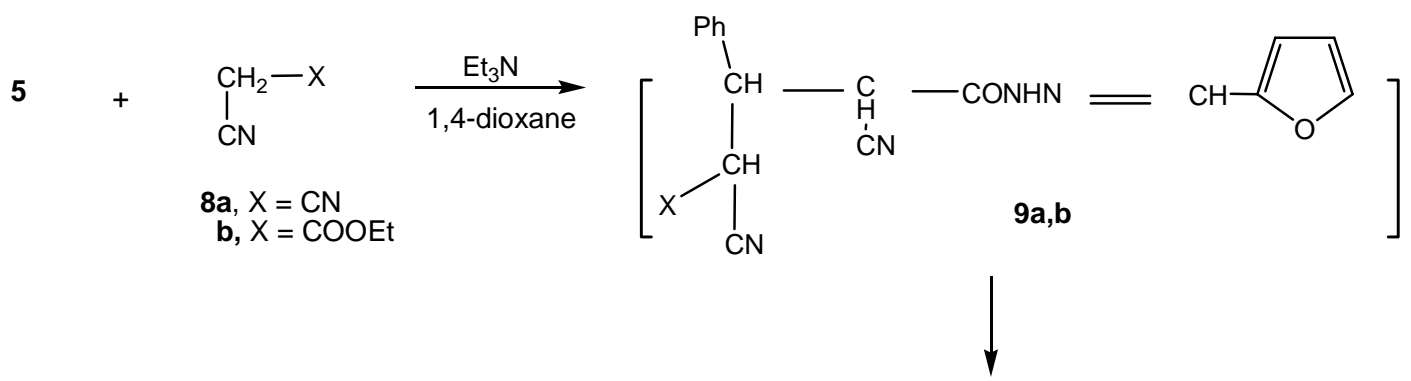<smiles>[X]c1c(N)c(-c2ccccc2)c(C#N)c(=O)n1N=C</smiles>

Scheme 1. Synthesis of compounds 3, 5, 7a,b and 10a,b.

$\left(\mathrm{D}_{2} \mathrm{O}\right.$-exchangeable) at $\delta 7.05(2 \mathrm{H}) \mathrm{ppm}$ equivalent to the $\mathrm{NH}_{2}$ group, a multiplet at $\delta 7.17-7.40(5 \mathrm{H})$ ppm indicating the phenyl protons and a singlet at $\delta 7.56(1 \mathrm{H})$ ppm corresponding to the $\mathrm{CH}$ group.

Further confirmation of the structures of $\mathbf{1 0 a}, \mathbf{b}$ was achieved through an alternative synthetic route involving the reaction of compound 3 with the cinnamonitrile reagents 11a,b to afford the same pyridone derivatives 10a, b (finger print IR, mp. and mixed mp.) with better yields $(95 \%, 98 \%)$ than in their formation by the reaction of compound 5 and either malononitrile (75\% yield) or ethyl cyanoacetate (74\% yield) (cf. Scheme 2). On the 
<smiles>CC(=O)NN=Cc1ccco1</smiles>

3
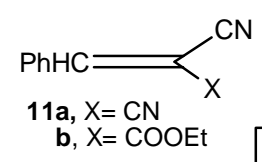<smiles>[X]C1CC1C(C#N)C(=O)N/N=C/c1ccco1</smiles>

$12 a, b$<smiles>[X]c1c(-c2ccccc2)c(C#N)c(=O)n(/N=C\c2ccco2)c1N</smiles>

$10 a, X=C N$ b, $X=$ COOEt<smiles>O=Cc1ccccc1O</smiles><smiles>[R]c1cc(C)c(C#N)c(=O)n1N=Cc1ccco1</smiles>

Scheme 2. Synthesis of compounds 10a,b, 14 and 16a,b.

other hand, compound 3 condensed with salicylaldehyde (13) to give the coumarin derivative 14. The analytical and spectral data of compound $\mathbf{1 4}$ are in agreement with the proposed structure. Thus, the ${ }^{1} \mathrm{H}$ NMR spectrum showed, a singlet at $\delta 6.95(1 \mathrm{H}) \mathrm{ppm}$ corresponding to coumarin $\mathrm{H}-4$, a singlet at $\delta 7.52(1 \mathrm{H}) \mathrm{ppm}$ corresponding to $\mathrm{CH}$ group, a multiplet at $\delta 6.52-7.88(3 \mathrm{H}) \mathrm{ppm}$ corresponding to furan protons, a multiplet at $\delta 7.33-7.55$ $(4 \mathrm{H}) \mathrm{ppm}$ corresponding to aromatic protons and a singlet $\left(\mathrm{D}_{2} \mathrm{O}\right.$-exchangeable) at $\delta 11.61(1 \mathrm{H}) \mathrm{ppm}$ corresponding to NH group. The reactivity of compound $\mathbf{3}$ towards 1,3-dicarbonyl compounds was studied to afford pyridine derivatives with wide range of biological activities thus, the reaction of 3 with either acetylacetone (15a) or ethyl acetoacetate (15b) gave the pyridine derivatives 16a and 16b, respectively (cf. Scheme 2). The structures of the latter products were established on the basis of analytical and spectral data. Thus, ${ }^{1} \mathrm{H}$ NMR spectrum of $\mathbf{1 6 b}$ showed a triplet at $\delta 1.35(3 \mathrm{H}) \mathrm{ppm}$ corresponding to $\mathrm{CH}_{3}$ group, a singlet at $\delta 2.34(3 \mathrm{H}) \mathrm{ppm}$ corresponding to $\mathrm{CH}_{3}$ group, a quartet at $\delta 4.22(2 \mathrm{H}) \mathrm{ppm}$ corresponding to $\mathrm{CH}_{2}$ group, a singlet at $\delta 5.79(1 \mathrm{H}) \mathrm{ppm}$ corresponding to pyridine proton, a singlet at $\delta 7.51(1 \mathrm{H}) \mathrm{ppm}$ corresponding to $\mathrm{CH}$ group and a multiplet at $\delta 6.52-7.88$ 
$(3 \mathrm{H}) \mathrm{ppm}$ corresponding to furan protons. On the other hand, we studied the reactivity of compound $\mathbf{3}$ with cyanomethylene reagents thus, the reaction of $\mathbf{3}$ with either malononitrile $\mathbf{( 8 a )}$ or ethyl cyanoacetate $\mathbf{( 8 b}$ ) gave the 2-pyridone derivatives 19a,b. The formation of the latter products took place via the intermediate formation of 17a,b (cf. Scheme 3). The ${ }^{1} \mathrm{H}$ NMR spectrum of $\mathbf{1 8 b}$ as an example showed a singlet at $\delta 5.80(1 \mathrm{H})$ ppm corresponding to the pyridine proton, a multiplet at $\delta 6.52$ $7.88(3 \mathrm{H}) \mathrm{ppm}$ corresponding to furan protons, a singlet $\left(\mathrm{D}_{2} \mathrm{O}\right.$-exchangeable) at $\delta 7.00(2 \mathrm{H}) \mathrm{ppm}$ corresponding to $\mathrm{NH}_{2}$ group, a singlet at $\delta 7.52(1 \mathrm{H}) \mathrm{ppm}$ corresponding to $\mathrm{CH}$ group, and a singlet $\left(\mathrm{D}_{2} \mathrm{O}\right.$-exchangeable $)$ at $\delta 12.56$ (1H) ppm corresponding to $\mathrm{OH}$ group.

The reaction of 3 with either acetophenone (19) or 4methoxy acetophenone (20) in ammonium acetate gave the Knoevenagel condensated products $\mathbf{2 1}$ and $\mathbf{2 2}$ respectively.

Next, we studied the reactivity of the active methylene group present in compound $\mathbf{3}$ towards diazonium salts. Thus, the reaction of compound $\mathbf{3}$ with aryldiazonium chlorides 23a-23d gave the phenylhydrazone derivatives 24a-24d. Analytical and spectral data of the latter products are all consistent with the proposed structures (see experimental section). Compound 24a reacted with ethyl cyanoacetate (8b) to give the pyridazine derivative $\mathbf{2 6}$. The reaction took place via the intermediate formation of 25 (cf. Scheme 3) On the other hand, the reaction of 24a with phenyl isothiocynate (27) gave the triazine derivative $\mathbf{2 9}$ via the intermediate formation of $\mathbf{2 8}$ (cf. Scheme 3). Analytical and spectral data of the latter product are in agreement with the proposed structure.

Recently our research group was involved through a comprehensive program dealing with studying the reaction of phenylisothiocyanate with active methylene reagents followed by heterocylization with $\alpha$-haloketones. The reactions lead to the formation of either thiazole or thiophene derivatives depending on the reaction conditions and the nature of the $\alpha$-halocarbonyl compounds.

In continuation of this program we report here the reaction of compound 3 with phenylisothiocyanate (27) in DMF solution containing $\mathrm{KOH}$ to form the intermediate potassium sulphide salt 30. The latter intermediate under went heterocyclization upon reaction with ethyl chloroacetate (31) to give the thiazole derivative 33. Formation of the latter product took place via the intermediate formation of 32. Similarly the reaction of $\mathbf{3 0}$ with $\alpha$ chloroacetone (34a) or phenacyl bromide (34b) gave the thiazole derivatives 35a and $\mathbf{3 5} \mathbf{b}$, respectively (Scheme 4). Analytical and spectral data are consistent with the proposed structures. Thus, ${ }^{1} \mathrm{H}$ NMR spectrum of $\mathbf{3 5 a}$ showed a singlet at $\delta 2.35(3 \mathrm{H}) \mathrm{ppm}$ corresponding to $\mathrm{CH}_{3}$ group, a singlet at $\delta 5.81(1 \mathrm{H}) \mathrm{ppm}$ corresponding to thiazole proton, a multiplet at $\delta 6.25-7.20(5 \mathrm{H}) \mathrm{ppm}$ corresponding to aromatic protons, a multiplet at $\delta 6.52$ $7.88(3 \mathrm{H}) \mathrm{ppm}$ corresponding to furan protons, a singlet at $\delta 7.52(1 \mathrm{H}) \mathrm{ppm}$ corresponding to $\mathrm{CH}$ group and a singlet $\left(\mathrm{D}_{2} \mathrm{O}\right.$-exchangeable $)$ at $\delta 11.62(1 \mathrm{H}) \mathrm{ppm}$ corresponding to $\mathrm{NH}$ group.

\subsection{Antitumor Activity}

\subsubsection{Material, Methods and Reagents}

Fetal bovine serum (FBS) and L-glutamine, were from Gibco Invitrogen Co. (Scotland, UK). RPMI-1640 medium was from Cambrex (New Jersey, USA). Dimethyl sulfoxide (DMSO), doxorubicin, penicillin, streptomycin and sulforhodamine B (SRB) were from Sigma Chemical Co. (Saint Louis, USA). Samples Stock solutions of selected compounds from 3-34a,b were prepared in DMSO and kept at $-20^{\circ} \mathrm{C}$. Appropriate dilutions of the compounds were freshly prepared just prior the assays. Final concentrations of DMSO did not interfere with the cell growth.

\subsubsection{Cell Cultures}

Three human tumor cell lines, MCF-7 (breast adenocarcinoma), NCI-H460 (non-small cell lung cancer), and SF-268 (CNS cancer) were used. MCF-7 was obtained from the European Collection of Cell Cultures (ECACC, Salisbury, UK) and NCI-H460 and SF-268 were kindly provided by the National Cancer Institute (NCI, Cairo, Egypt). They grow as monolayer and routinely maintained in RPMI-1640 medium supplemented with 5\% heat inactivated FBS, $2 \mathrm{mM}$ glutamine and antibiotics (penicillin $100 \mu / \mathrm{mL}$, streptomycin $100 \mu \mathrm{g} / \mathrm{mL}$ ), at $37^{\circ} \mathrm{C}$ in a humidified atmosphere containing $5 \% \mathrm{CO}_{2}$. Exponentially growing cells were obtained by plating $1.5 \times$ $10^{5}$ cells $/ \mathrm{mL}$ for MCF-7 and SF-268 and $0.75 \times 10^{4}$ cells/ $\mathrm{mL}$ for NCI-H460, followed by $24 \mathrm{~h}$ of incubation. The effect of the vehicle solvent (DMSO) on the growth of these cell lines was evaluated in all the experiments by exposing untreated control cells to the maximum concentration $(0.5 \%)$ of DMSO used in each assay.

\subsubsection{Effect on the Growth of Human Tumor Cell Lines}

The effect of selected compounds from the newly synthesized products 3-35a, $\mathbf{b}$ was evaluated on the in vitro growth of three human tumor cell lines representing different tumor types, namely, breast adenocarcinoma (MCF7), non-small cell lung cancer (NCI-H460) and CNS cancer (SF-268), after a continuous exposure of $48 \mathrm{~h}$. the evaluations of the newly synthesized products were measured using doxorubicin as the positive control and the results are summarized in Table $\mathbf{1 .}$

All the compounds were able to inhibit the growth of the human tumor cell lines in a dose-dependent manner 


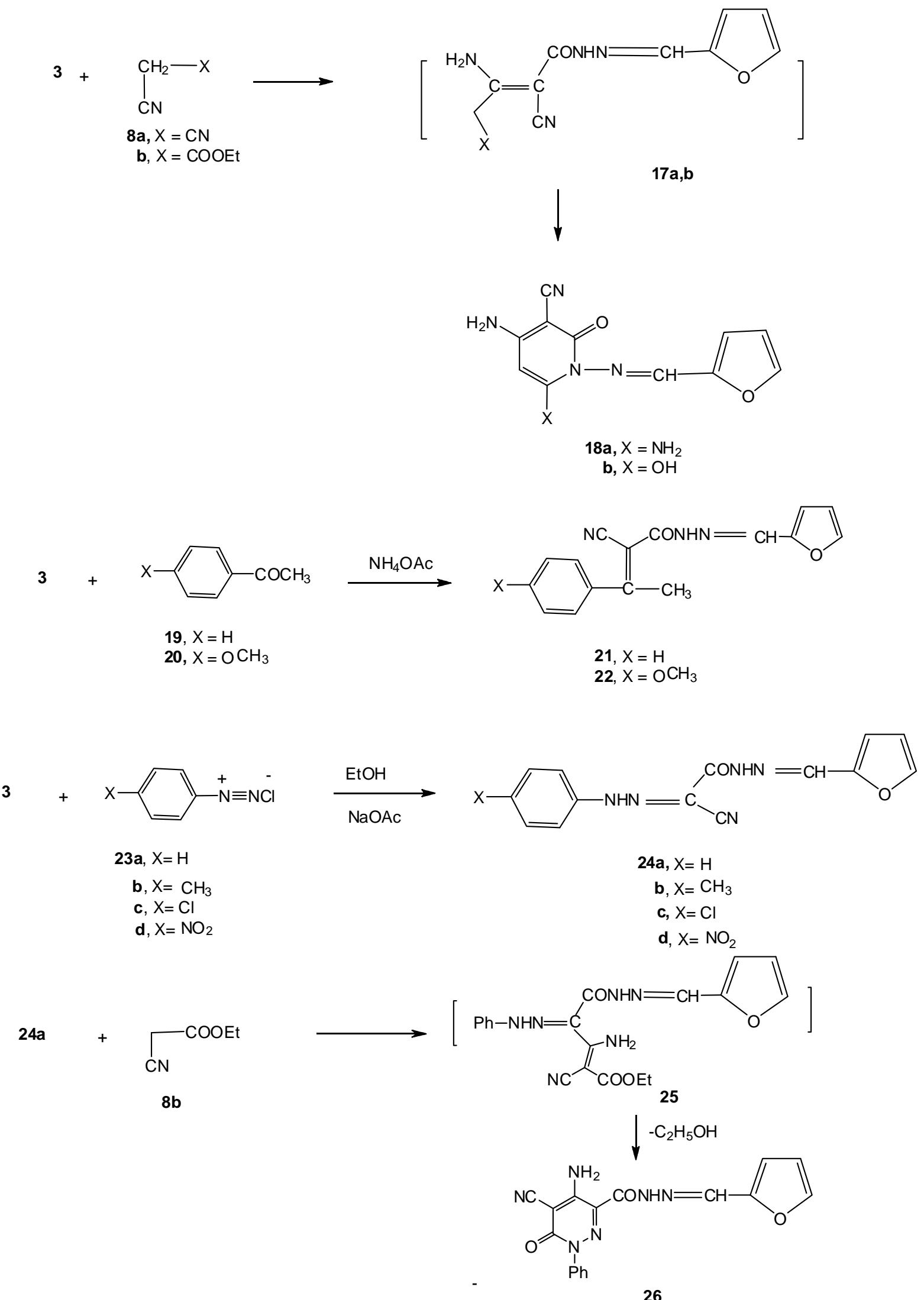

Scheme 3. Synthesis of compounds 18a, 18b, 21, 22, 24a-24d and 26. 


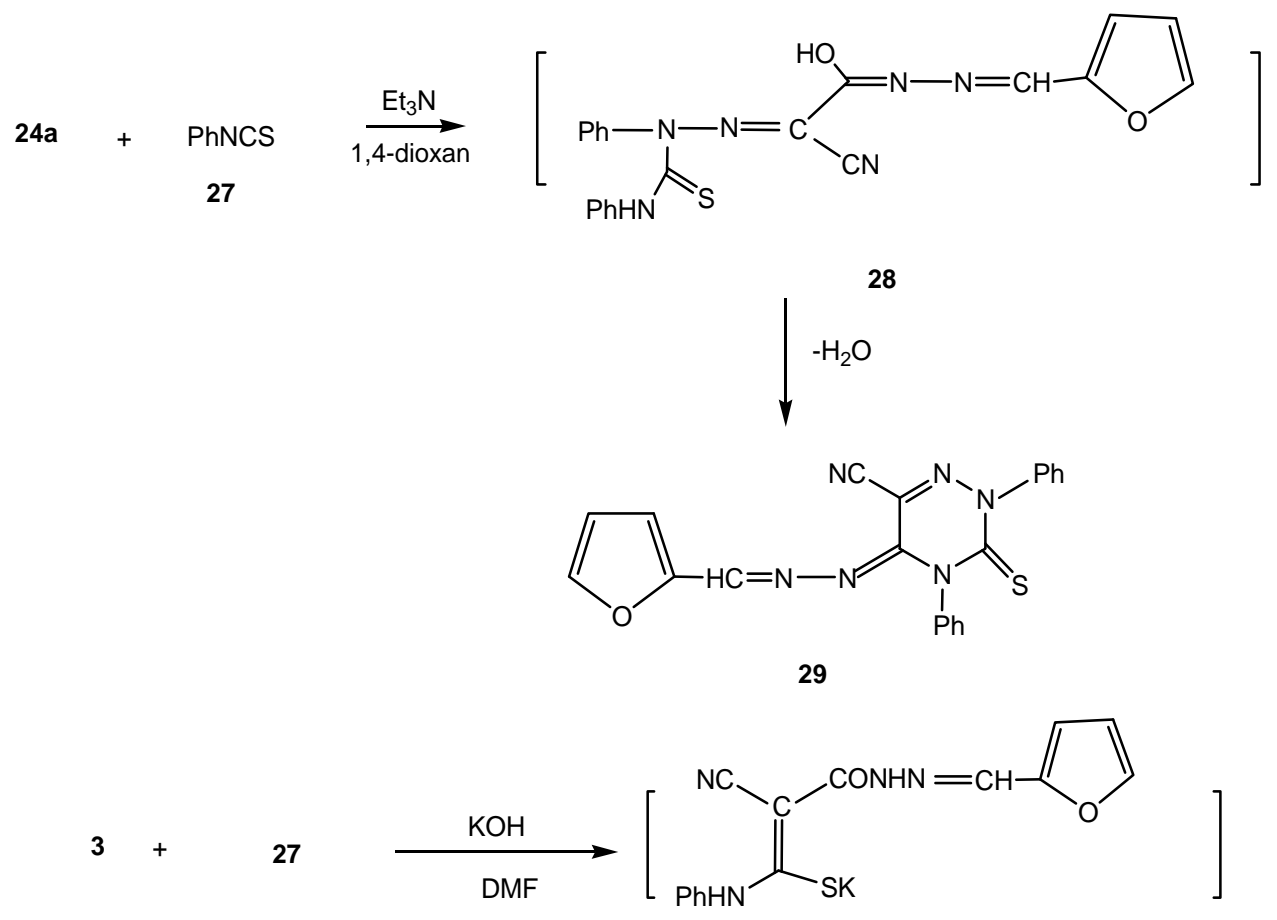

30<smiles>[R]C1=CS/C(=C(/C#N)C(=O)N/N=C/c2ccco2)N1c1[R17]:[R]([R]#P)ccc1</smiles>

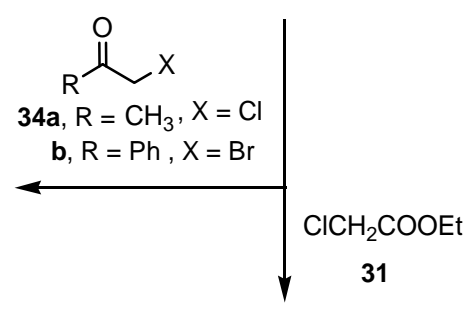<smiles>N#CC(C(=O)N=CC1=CCCO1)=C1SC=C(O)N1c1ccccc1</smiles><smiles>CCOC(=O)CSC(N[PH2+]CC)=C(C#N)C(=O)N=Cc1ccco1</smiles>

Scheme 4. Synthesis of compounds 29, 30, 33 and 35a,b.

(data not shown). The pyrazole derivative $\mathbf{7 b}$ and the coumarine derivative $\mathbf{1 4}$ showed the best results, exhibiting an equivalent potency in all the three tumor cell lines which is still much lower than the gram positive control doxorubicin. On the other hand, compounds $3,7 \mathbf{a}$, 10a, 18a, 18b, 21, 22, 24c, 26 and 33 showed moderated growth inhibitory effect. Comparing the activities of 18a and $\mathbf{1 8 b}$ it is observed that the hydroxyl group in $\mathbf{1 8 b}$ presents a stronger growth inhibitory effect than the amino substituent in 18a although the results in NCIH460 cell line are comparable. It is clear from Table 1 that some compounds like $\mathbf{1 0 b}, \mathbf{1 6 a}, \mathbf{b}$ and $\mathbf{3 5 b}$ showed very low activity towards certain cell line, MCF-7, and moderate activity towards other cell lines.

Results are given in concentrations that were able to cause $50 \%$ of cell growth inhibition $\left(\mathrm{GI}_{50}\right)$ after a continuous exposure of $48 \mathrm{~h}$ and show means \pm SEM of three-independent experiments performed in duplicate.

\section{Conclusion}

In conclusion, we have developed efficient methods for the synthesis of new hydrazide-hydrazone derivatives via 
Table 1. Effect of the newly synthesized products on the growth of three human tumor cell lines.

\begin{tabular}{|c|c|c|c|}
\hline \multirow{2}{*}{ Compound } & \multicolumn{3}{|c|}{$\mathrm{GI}_{50}(\mu \mathrm{mol} / \mathrm{L})$} \\
\hline & MCF-7 & NCI-H460 & SF-268 \\
\hline 3 & $20.0 \pm 0.2$ & $30.6 \pm 1.4$ & $38.4 \pm 0.6$ \\
\hline 5 & $4.1 \pm 0.7$ & $2.2 \pm 0.8$ & $2.4 \pm 1.8$ \\
\hline $7 a$ & $36.6 \pm 12.2$ & $30.6 \pm 8.6$ & $60.4 \pm 14.8$ \\
\hline $7 b$ & $0.04 \pm 0.01$ & $0.01 \pm 0.008$ & $0.03 \pm 0.01$ \\
\hline $10 \mathrm{a}$ & $20.2 \pm 0.4$ & $26.3 \pm 0.8$ & $30 \pm 0.8$ \\
\hline $10 \mathrm{~b}$ & $70.6 \pm 16.9$ & $38.9 \pm 10.8$ & $50.8 \pm 8.6$ \\
\hline 14 & $0.1 \pm 0.07$ & $0.02 \pm 0.008$ & $1.2 \pm 0.2$ \\
\hline $16 \mathrm{a}$ & $42.7 \pm 17.5$ & $20.2 \pm 12.8$ & $50.0 \pm 9.01$ \\
\hline $16 \mathrm{~b}$ & $50.1 \pm 0.7$ & $23.2 \pm 4.8$ & $18.4 \pm 1.8$ \\
\hline $18 \mathrm{a}$ & $22.7 \pm 17.5$ & $36.2 \pm 12.8$ & $40.0 \pm 9.01$ \\
\hline $18 \mathrm{~b}$ & $12.7 \pm 17.5$ & $40.2 \pm 12.8$ & $50.0 \pm 9.01$ \\
\hline 21 & $10.8 \pm 0.6$ & $12.5 \pm 0.8$ & $16.7 \pm 1.6$ \\
\hline 22 & $20.0 \pm 0.2$ & $30.6 \pm 1.4$ & $38.4 \pm 0.6$ \\
\hline $24 a$ & $35.0 \pm 1.8$ & $44.0 \pm 0.8$ & $20.5 \pm 1.1$ \\
\hline $24 b$ & $11.9 \pm 0.6$ & $14.1 \pm 0.6$ & $20.3 \pm 0.5$ \\
\hline $24 c$ & $18.0 \pm 0.6$ & $20.0 \pm 0.4$ & $30.5 \pm 8.0$ \\
\hline 24d & $10.8 \pm 0.6$ & $12.5 \pm 0.8$ & $16.7 \pm 1.6$ \\
\hline 26 & $22.4 \pm 10.2$ & $25.1 \pm 0.8$ & $16.9 \pm 4.8$ \\
\hline 29 & $33.2 \pm 0.6$ & $15.3 \pm 1.4$ & $20.3 \pm 1.5$ \\
\hline 33 & $20.0 \pm 0.4$ & $24.3 \pm 0.8$ & $32 \pm 0.8$ \\
\hline $35 \mathrm{a}$ & $31.0 \pm 1.8$ & $40.0 \pm 0.8$ & $18.5 \pm 1.1$ \\
\hline $35 \mathrm{~b}$ & $70.6 \pm 16.9$ & $38.9 \pm 10.8$ & $50.8 \pm 8.6$ \\
\hline doxorubicin & $0.04 \pm 0.008$ & $0.09 \pm 0.008$ & $0.09 \pm 0.007$ \\
\hline
\end{tabular}

the reaction of cyanoacetylhydrazine with furan-2-aldehyde. The produced product underwent a series of heterocyclizations. Their antitumor evaluations through the three cancer cell lines showed that compounds $\mathbf{7 b}$ and $\mathbf{1 4}$ showed the maximum inhibitory effect among the tested compounds.

\section{Experimental}

All melting points were determined in open capillaries and are uncorrected. Elemental analyses were performed on a Yanaco CHNS Corder elemental analyzer (Japan). IR spectra were measured using $\mathrm{KBr}$ discs on a Pye Unicam SP-1000 spectrophotometer. ${ }^{1} \mathrm{H}$ NMR spectra were measured on a Varian EM 390 - $200 \mathrm{MHz}$ instrument in $\mathrm{CD}_{3} \mathrm{SOCD}_{3}$ as solvent using TMS as internal standard and chemical shifts are expressed as $\delta$ ppm. Mass spectra were recorded on Kratos $(75 \mathrm{eV})$ MS equipment (Germany). Elemental analyses were carried out by the Micro-analytical Data Center at Cairo University and were performed on Vario EL III Elemental CHNS analyzer.

2-Cyano- $N$-(furan-2-ylmethylene)acetohydrazide (3). To a solution of compound $1(0.099 \mathrm{~g}, 1.0 \mathrm{mmol})$ in 1,4dioxane $(30 \mathrm{~mL})$ and furfural (2) $(0.09 \mathrm{~g}, 1.0 \mathrm{mmol})$ were added. The reaction mixture was heated under reflux for $3 \mathrm{hr}$, then was left until the reaction mixture be cooled. The formed solid product was collected by filtration. Yellow crystals from 1,4-dioxane, 98\% $(0.097 \mathrm{~g})$ yield; mp. $190^{\circ} \mathrm{C}-192^{\circ} \mathrm{C}$, MS: $\mathrm{m} / \mathrm{z}=177\left(\mathrm{M}^{+}\right)$, IR $(\mathrm{KBr})$ : $\mathrm{v} / \mathrm{cm}^{-1}=3200(\mathrm{NH}), 3059\left(\mathrm{CH}_{\mathrm{Ar}}\right), 2198(\mathrm{CN}), 1690$ (CO), $1550(\mathrm{C}=\mathrm{N}), 750(\mathrm{C}-\mathrm{O}),{ }^{1} \mathrm{H}$ NMR (DMSO-d $\left.{ }_{6}\right): \delta=$ $6.60\left(\mathrm{~s}, 2 \mathrm{H}, \mathrm{CH}_{2}\right), 6.52-7.88(\mathrm{~m}, 3 \mathrm{H}$, furan- $\mathrm{CH}), 7.54(\mathrm{~s}$, $1 \mathrm{H}, \mathrm{CH}), 11.62\left(\mathrm{~s}, 1 \mathrm{H}, \mathrm{NH}, \mathrm{D}_{2} \mathrm{O}\right.$-exchangeable). Analysis Calcd for $\mathrm{C}_{8} \mathrm{H}_{7} \mathrm{~N}_{3} \mathrm{O}_{2}$ (177.16): C, 54.24; H, 3.98; N, $23.72 \%$. Found: C, $54.11 ; \mathrm{H}, 4.25$; N, $23.59 \%$.

2-Cyano- $N$-(furan-2-ylmethylene)-3-phenylacryloh ydrazide (5). To a solution of compound $3(0.177 \mathrm{~g}, 1.0$ $\mathrm{mmol})$ in ethanol $(30 \mathrm{~mL})$, piperidine (3 drops) and benzaldehyde (4) $(0.10 \mathrm{~g}, 1.0 \mathrm{mmol})$ were added. The reaction mixture was heated under reflux for $3 \mathrm{~h}$, and then poured on an ice/water mixture containing a few drops of hydrochloric acid. The formed solid product was collected by filtration.

Yellow crystals from 1,4-dioxane, 95\% (0.168 g) yield; mp. $140^{\circ} \mathrm{C}-142^{\circ} \mathrm{C}, \mathrm{MS}: \mathrm{m} / \mathrm{z}=265\left(\mathrm{M}^{+}\right)$, IR $(\mathrm{KBr})$ : $\mathrm{v} / \mathrm{cm}^{-1}=3200(\mathrm{NH}), 3159\left(\mathrm{CH}_{\mathrm{Ar}}\right), 2200,1680(2 \mathrm{CN})$, $1580(\mathrm{C}=\mathrm{C}), 1550(\mathrm{C}=\mathrm{N}), 780(\mathrm{C}-\mathrm{O}),{ }^{1} \mathrm{H}$ NMR $\left(\right.$ DMSO- $\left._{6}\right): \delta=6.52-7.88(\mathrm{~m}, 3 \mathrm{H}$, furan-CH), $7.84(\mathrm{~s}$, $1 \mathrm{H}, \mathrm{CH}), 7.33-7.60\left(\mathrm{~m}, 5 \mathrm{H}, \mathrm{H}_{\mathrm{Ar}}\right), 8.45(\mathrm{~s}, 1 \mathrm{H}, \mathrm{CH})$, 11.61 (s, $1 \mathrm{H}, \mathrm{NH}, \mathrm{D}_{2} \mathrm{O}$-exchangeable). Analysis Calcd for $\mathrm{C}_{15} \mathrm{H}_{11} \mathrm{~N}_{3} \mathrm{O}_{2}$ (265.27): C, 67.92; H, 4.18; N, 15.84\%. Found: C, 68.26; H, 4.28; N, $15.93 \%$.

Synthesis of pyrazole derivatives $7 \mathbf{a}, \mathbf{b}$.

General procedure: To a solution of compound 5 $(0.265 \mathrm{~g}, 1.0 \mathrm{mmol})$ in 1,4-dioxane $(25 \mathrm{~mL})$ and dimethylformamide $(10 \mathrm{~mL})$, either hydrazine hydrate (6a) $(0.05 \mathrm{~g}, 1.0 \mathrm{mmol})$ or phenylhydrazine (6b) $(0.10 \mathrm{~g}, 1.0$ mmol) was added. The reaction mixture in each case was heated under reflux for $3 \mathrm{~h}$, and then poured on an ice/ water mixture containing a few drops of hydrochloric acid. The formed solid product in each case was collected by filtration.

3-Amino- $N$-(furan-2-ylmethylene)-5-phenyl-1 $\mathrm{H}$-pyr azole-4carbohyd-razide (7a). Brown crystals from 1,4dioxane/dimethylformamide mixture, $87 \%(0.230 \mathrm{~g})$ yield, mp. $250^{\circ} \mathrm{C}-252^{\circ} \mathrm{C}, \mathrm{IR}(\mathrm{KBr}): \mathrm{v} / \mathrm{cm}^{-1}=3380\left(\mathrm{NH}_{2}\right)$, 3400, $3320(2 \mathrm{NH}), 3200\left(\mathrm{CH}_{\mathrm{Ar}}\right), 1680(\mathrm{CO}), 1560(\mathrm{C}=\mathrm{N})$, 780 (C-O), ${ }^{1} \mathrm{H}$ NMR (DMSO-d $\left.{ }_{6}\right): \delta=6.52-7.88(\mathrm{~m}, 3 \mathrm{H}$, furan- $\mathrm{CH}), 7.00,7.20\left(\mathrm{~s}, 2 \mathrm{H}, \mathrm{NH}_{2}, \mathrm{D}_{2} \mathrm{O}\right.$-exchangeable), 
$7.41-7.79\left(\mathrm{~m}, 5 \mathrm{H}, \mathrm{H}_{\mathrm{Ar}}\right), 7.55(\mathrm{~s}, 1 \mathrm{H}, \mathrm{CH}), 11.62(\mathrm{~s}, 1 \mathrm{H}$, $\mathrm{NH}, \mathrm{D}_{2} \mathrm{O}$-exchangeable), 13.7 (s, $1 \mathrm{H}$, pyrazole- $\mathrm{NH}, \mathrm{D}_{2} \mathrm{O}-$ exchangeable). Analysis Calcd for $\mathrm{C}_{15} \mathrm{H}_{13} \mathrm{~N}_{5} \mathrm{O}_{2}$ (295.30): C, 61.01; H, 4.44; N, 23.72 \%. Found: C, 61.34; H, 4.62; $\mathrm{N}, 23.91 \%$.

3-Amino-1,5-diphenyl- $N$-(furan-2-ylmethylene)-1 $H$ pyrazole-4-carb-ohydrazide (7b). Yellowish brown crystals from 1,4-dioxane/dimethylformamide mixture, $90 \%(0.238 \mathrm{~g})$ yield, $\mathrm{mp} .148^{\circ} \mathrm{C}-150^{\circ} \mathrm{C}, \mathrm{IR}(\mathrm{KBr}): \mathrm{v} / \mathrm{cm}^{-1}$ $=3370\left(\mathrm{NH}_{2}\right), 3250(\mathrm{NH}), 2690-3400\left(\mathrm{CH}_{\mathrm{Ar}}\right), 1665$ (CO), $1600(\mathrm{C}=\mathrm{N}), 775(\mathrm{C}-\mathrm{O}),{ }^{1} \mathrm{H}$ NMR (DMSO-d $\left.{ }_{6}\right): \delta=$ $6.52-7.88(\mathrm{~m}, 3 \mathrm{H}$, furan- $\mathrm{CH}), 7.41-7.79\left(\mathrm{~m}, 10 \mathrm{H}, \mathrm{H}_{\mathrm{Ar}}\right)$, 7.00, $7.20\left(\mathrm{~s}, 2 \mathrm{H}, \mathrm{NH}_{2}, \mathrm{D}_{2} \mathrm{O}\right.$-exchangeable), $7.55(\mathrm{~s}, 1 \mathrm{H}$, $\mathrm{CH}), 11.62\left(\mathrm{~s}, 1 \mathrm{H}, \mathrm{NH}, \mathrm{D}_{2} \mathrm{O}\right.$-exchangeable). Analysis Calcd for $\mathrm{C}_{21} \mathrm{H}_{17} \mathrm{~N}_{5} \mathrm{O}_{2}$ (371.39): C, 67.91; H, 4.61; N, $18.86 \%$. Found: C, $68.20 ; \mathrm{H}, 4.81 ; \mathrm{N}, 19.02 \%$.

Synthesis of pyridine derivatives 10a,b.

Method A: To a solution of compound $5(0.265 \mathrm{~g}, 1.0$ $\mathrm{mmol})$ either malononitrile (8a) $(0.06 \mathrm{~g}, 1.0 \mathrm{mmol})$ or ethyl cyanoacetate (8b) $(0.11 \mathrm{~g}, 1.0 \mathrm{mmol})$ was added in 1,4-dioxane $(30 \mathrm{~mL})$ containing triethylamine (3 drops). The reaction mixture was heated under reflux for $3 \mathrm{~h}$. then poured on an ice/water mixture containing a few drops of hydrochloric acid. The solid product formed in each case was collected by filtration.

Method B: To a solution of compound $3(0.177 \mathrm{~g}, 1.0$ mmol) either benzylidene malononitrile (11a) $(0.154 \mathrm{~g}$, $1.0 \mathrm{mmol})$ or benzylidene ethyl cyanoacetate (11b) $(0.201 \mathrm{~g}, 1.0 \mathrm{mmol})$ was added in 1,4-dioxane $(30 \mathrm{~mL})$ containing triethylamine ( 3 drops). The reaction mixture was heated under reflux for $3 \mathrm{~h}$, and then poured on an ice/water mixture containing a few drops of hydrochloric acid. The solid product formed in each case was collected by filtration.

6-Amino-1-(furan-2-ylmethyleneamine)-2-oxo-4-phenyl-1,2-dihydro-pyridine-3,5-dicarbonitrile (10a). Dark red crystals from 1,4-dioxane, yield [75\% $(0.198 \mathrm{~g})$, method A; $95 \%(0.168 \mathrm{~g})$, method B], mp. $190^{\circ} \mathrm{C}$ $192^{\circ} \mathrm{C}$, MS: $\mathrm{m} / \mathrm{z}=329\left(\mathrm{M}^{+}\right), \mathrm{IR}(\mathrm{KBr}): \mathrm{v} / \mathrm{cm}^{-1}=3300$ $\left(\mathrm{CH}_{\mathrm{Ar}}\right), 3220\left(\mathrm{NH}_{2}\right), 2350,2480(2 \mathrm{CN}), 1665(\mathrm{CO}), 1580$ $(\mathrm{C}=\mathrm{N}),{ }^{1} \mathrm{H}$ NMR $\left(\mathrm{DMSO}_{6}\right): \delta=6.52-7.88(\mathrm{~m}, 3 \mathrm{H}$, furan- $\mathrm{CH}), 7.05\left(\mathrm{~s}, 2 \mathrm{H}, \mathrm{NH}_{2}, \mathrm{D}_{2} \mathrm{O}\right.$-exchangeable), 7.17 $7.60\left(\mathrm{~m}, 5 \mathrm{H}, \mathrm{H}_{\mathrm{Ar}}\right), 7.56(\mathrm{~s}, 1 \mathrm{H}, \mathrm{CH})$. Analysis Calcd for $\mathrm{C}_{18} \mathrm{H}_{11} \mathrm{~N}_{5} \mathrm{O}_{2}$ (329.31): C, 65.65; H, 3.37; N, 21.27\%. Found: C, 65.73; H, 3.59; N, 21.38\%.

Ethyl-2-amino-5-cyano-1-(furan-2-ylmethylene-ami n)-6-oxo-4-phen-yl-1,6-dihydropyridine-3-carboxylate (10b). Brown crystals from 1,4-dioxane, yield $74 \%$ (0.196 g), method A; 98\% (0.173 g), method B], mp. $180^{\circ} \mathrm{C}-182^{\circ} \mathrm{C}, \mathrm{MS}: \mathrm{m} / \mathrm{z}=376\left(\mathrm{M}^{+}\right), \mathrm{IR}(\mathrm{KBr}): \mathrm{v} / \mathrm{cm}^{-1}=$ $3210\left(\mathrm{NH}_{2}\right), 3180-3050\left(\mathrm{CH}_{\mathrm{Ar}}\right), 2380(\mathrm{CN}), 1660(\mathrm{CO})$, $1565(\mathrm{C}=\mathrm{N}), 1190(\mathrm{C}-\mathrm{O}),{ }^{1} \mathrm{H}$ NMR $\left(\mathrm{DMSO}^{-} \mathrm{d}_{6}\right): \delta=1.29$ (t, $\left.3 \mathrm{H}, \mathrm{J}=7 \mathrm{~Hz}, \mathrm{CH}_{3}\right), 4.24\left(\mathrm{q}, 2 \mathrm{H}, \mathrm{J}=7 \mathrm{~Hz}, \mathrm{CH}_{2}\right), 6.52$ $7.88(\mathrm{~m}, 3 \mathrm{H}$, furan- $\mathrm{CH}), 7.05\left(\mathrm{~s}, 2 \mathrm{H}, \mathrm{NH}_{2}, \mathrm{D}_{2} \mathrm{O}\right.$-excha- ngeable), 7.17 - $7.40\left(\mathrm{~m}, 5 \mathrm{H}, \mathrm{H}_{\mathrm{Ar}}\right), 7.56(\mathrm{~s}, 1 \mathrm{H}, \mathrm{CH})$. Analysis Calcd for $\mathrm{C}_{20} \mathrm{H}_{16} \mathrm{~N}_{4} \mathrm{O}_{4}$ (376.37): C, 63.82; H, 4.28; N, 14.89\%. Found: C, 63.90; H, 4.41; N, 14.58\%.

N'-(Furan-2-ylmethylene)-2-oxo-2H-coumarin-3-car bohydrazide (14). To a solution of compound $3(0.177 \mathrm{~g}$, $1.0 \mathrm{mmol})$ in 1,4-dioxane $(20 \mathrm{~mL})$, piperidine (3 drops) and salicaldhyde (13) $(0.12 \mathrm{~g}, 1.0 \mathrm{mmol})$ were added. The reaction mixture was heated under reflux for $2 \mathrm{~h}$, and then poured on an ice/water mixture containing a few drops of hydrochloric acid. The solid product was collected by filtration. Yellow crystals from dimethylformamide, $95 \%(0.168 \mathrm{~g})$ yield, $\mathrm{mp} .280^{\circ} \mathrm{C}-282^{\circ} \mathrm{C}, \mathrm{MS}: \mathrm{m} / \mathrm{z}$ $=282\left(\mathrm{M}^{+}\right)$, IR $(\mathrm{KBr}): \mathrm{v} / \mathrm{cm}^{-1}=3400\left(\mathrm{CH}_{\mathrm{Ar}}\right), 3100(\mathrm{NH})$, 1700, $1600(2 \mathrm{CO}), 1550(\mathrm{C}=\mathrm{N}),{ }^{1} \mathrm{H}$ NMR $\left(\mathrm{DMSO}-\mathrm{d}_{6}\right): \delta$ $=6.95\left(\mathrm{~s}, 1 \mathrm{H}\right.$, coumarin $\left.\mathrm{C}_{4}-\mathrm{H}\right), 7.52(\mathrm{~s}, 1 \mathrm{H}, \mathrm{CH}), 6.52$ $7.88(\mathrm{~m}, 3 \mathrm{H}$, furan- $\mathrm{CH}), 7.33-7.55\left(\mathrm{~m}, 4 \mathrm{H}, \mathrm{H}_{\mathrm{Ar}}\right), 11.61$ (s, $1 \mathrm{H}, \mathrm{NH}, \mathrm{D}_{2} \mathrm{O}$-exchangeable). Analysis Calcd for $\mathrm{C}_{15} \mathrm{H}_{10} \mathrm{~N}_{2} \mathrm{O}_{4}$ (282.25): C, 63.83; H, 3.57; N, 9.92\%. Found: C, 63.97; H, 3.73; N, 10.31\%.

Synthesis of pyridine derivatives $16 \mathrm{a}, \mathrm{b}$.

General procedure: To a solution of compound 3 $(0.177 \mathrm{~g}, 1.0 \mathrm{mmol})$ either acetylacetone (15a) $(0.1 \mathrm{~g}, 1.0$ $\mathrm{mmol})$ or ethyl acetoacetate $\mathbf{( 1 5 b )}(0.13 \mathrm{~g}, 1.0 \mathrm{mmol})$ was added in 1,4-dioxane $(25 \mathrm{~mL})$ containing piperidine ( 3 drops). The reaction mixture was heated under reflux for $5 \mathrm{~h}$, and then poured on an ice/water mixture containing a few drops of hydrochloric acid. The solid product formed in each case was collected by filtration.

4,6-Dimethyl-1-(furan-2-ylmethyleneamino)-2-oxo1,2-dihydropyrid-ine-3-carbonitrile (16a). Brown crystals from 1,4-dioxane, $85 \%(0.150 \mathrm{~g})$ yield, $\mathrm{mp} .80^{\circ} \mathrm{C}-$ $82^{\circ} \mathrm{C}, \mathrm{MS}: \mathrm{m} / \mathrm{z}=241\left(\mathrm{M}^{+}\right), \mathrm{IR}(\mathrm{KBr}): \mathrm{v} / \mathrm{cm}^{-1}=2942$, $2932\left(2 \mathrm{CH}_{3}\right), 2900\left(\mathrm{CH}_{\mathrm{Ar}}\right), 2300(\mathrm{CN}) 1620(\mathrm{CO}), 1565$ $(\mathrm{C}=\mathrm{N}),{ }^{1} \mathrm{H}$ NMR $\left(\right.$ DMSO- $\left._{6}\right): \delta=2.22,2.34(2 \mathrm{~s}, 6 \mathrm{H}$, $\left.2 \mathrm{CH}_{3}\right), 5.78(\mathrm{~s}, 1 \mathrm{H}$, pyridine $\mathrm{H}-3), 7.52(\mathrm{~s}, 1 \mathrm{H}, \mathrm{CH}=\mathrm{N})$, $6.52-7.88(\mathrm{~m}, 3 \mathrm{H}$, furan-CH). Analysis Calcd for $\mathrm{C}_{13} \mathrm{H}_{11} \mathrm{~N}_{3} \mathrm{O}_{2}$ (241.25): C, 64.72; H, 4.60; N, $17.42 \%$. Found: C, 64.82; H, 4.44; N, 17.72\%.

6-Ethoxy-1-(furan-2-ylmethyleneamino)-4-methyl-2oxo-1,2-dihydro-pyridine-3-carbonitrile (16b). Reddish brown crystals from dimethylformamide, $82 \%(0.145 \mathrm{~g})$ yield, $\mathrm{mp} .240^{\circ} \mathrm{C}-242^{\circ} \mathrm{C}, \mathrm{MS}: \mathrm{m} / \mathrm{z}=271\left(\mathrm{M}^{+}\right), \mathrm{IR}(\mathrm{KBr})$ : $\mathrm{v} / \mathrm{cm}^{-1}=2940\left(\mathrm{CH}_{3}\right), 2910\left(\mathrm{CH}_{\mathrm{Al}}\right), 2900\left(\mathrm{CH}_{\mathrm{Ar}}\right), 2280$ $(\mathrm{CN}), 1630(\mathrm{CO}), 1560(\mathrm{C}=\mathrm{N}), 1070(\mathrm{C}-\mathrm{O}),{ }^{1} \mathrm{H}$ NMR $\left(\mathrm{DMSO}_{6} \mathrm{~d}_{6}\right): \delta=1.35\left(\mathrm{t}, 3 \mathrm{H}, \mathrm{CH}_{3}\right), 2.34\left(\mathrm{~s}, 3 \mathrm{H}, \mathrm{CH}_{3}\right)$, $4.22\left(\mathrm{q}, 2 \mathrm{H}, \mathrm{CH}_{2}\right), 5.79(\mathrm{~s}, 1 \mathrm{H}$, pyridine $\mathrm{H}), 7.51(\mathrm{~s}, 1 \mathrm{H}$, $\mathrm{CH}=\mathrm{N}), 6.52-7.88(\mathrm{~m}, 3 \mathrm{H}$ furan-CH). Analysis Calcd for $\mathrm{C}_{14} \mathrm{H}_{13} \mathrm{~N}_{3} \mathrm{O}_{3}$ (271.27): $\mathrm{C}, 61.99 ; \mathrm{H}, 4.83 ; \mathrm{N}, 15.49 \%$. Found: C, 62.28; H, 4.93; N, 15.72\%.

Synthesis of pyridine derivatives 18a,b.

General procedure: To a solution of compound $\mathbf{3}$ $(0.177 \mathrm{~g}, 1.0 \mathrm{mmol})$ either malononitrile (8a) $(0.06 \mathrm{~g}, 1.0$ $\mathrm{mmol})$ or ethyl cyanoacetate $\mathbf{( 8 b )}(0.11 \mathrm{~g}, 1.0 \mathrm{mmol})$ was added in 1,4-dioxane $(25 \mathrm{~mL})$ containing triethylamine 
(4 drops). The reaction mixture was heated under reflux for $3 \mathrm{~h}$, and then poured on an ice/water mixture containing a few drops of hydrochloric acid. The solid product formed in each case was collected by filtration.

4,6-Diamino-1-(furan-2-ylmethyleneamino)-2-oxo-1, 2-dihydropyrid-ine-3-carbonitrile (18a). Red crystals from dimethylformamide, 95\% (0.168 g) yield, mp. $250^{\circ} \mathrm{C}-252^{\circ} \mathrm{C}, \mathrm{MS}: \mathrm{m} / \mathrm{z}=243\left(\mathrm{M}^{+}\right)$, IR $(\mathrm{KBr}): \mathrm{v} / \mathrm{cm}^{-1}=$ 3150, $3200\left(2 \mathrm{NH}_{2}\right), 2150(\mathrm{CN}), 1650(\mathrm{CO}), 1550(\mathrm{C}=\mathrm{N})$, ${ }^{1} \mathrm{H}$ NMR $\left(\mathrm{DMSO}-\mathrm{d}_{6}\right): \delta=5.80(\mathrm{~s}, 1 \mathrm{H}$, pyridine $\mathrm{CH}), 6.53$ - $7.88\left(\mathrm{~m}, 3 \mathrm{H}\right.$, furan-CH), $7.00,7.19\left(2 \mathrm{~s}, 4 \mathrm{H}, 2 \mathrm{NH}_{2}\right.$, $\mathrm{D}_{2} \mathrm{O}$-exchangeable), $7.51(\mathrm{~s}, 1 \mathrm{H}, \mathrm{CH})$. Analysis Calcd for $\mathrm{C}_{11} \mathrm{H}_{9} \mathrm{~N}_{5} \mathrm{O}_{2}$ (243.22): C, 54.32; H, 3.73; N, 28.79\%. Found: C, 54.52; H, 3.93; N, 28.83\%.

4-Amino-1-(furan-2-ylmethyleneamino)-6-hydroxy2-oxo-1,2-dihydr-opyridine-3-carbonitrile (18b). Yellow crystals from 1,4-dioxane, $98 \%(0.173 \mathrm{~g})$ yield, $\mathrm{mp} .80^{\circ} \mathrm{C}$ - $83^{\circ} \mathrm{C}, \mathrm{MS}: \mathrm{m} / \mathrm{z}=244\left(\mathrm{M}^{+}\right), \mathrm{IR}(\mathrm{KBr}): \mathrm{v} / \mathrm{cm}^{-1}=3450$ $(\mathrm{OH}), 3150\left(\mathrm{NH}_{2}\right), 3050\left(\mathrm{CH}_{\mathrm{Al}}\right), 2130(\mathrm{CN}), 1650(\mathrm{CO})$, $1550(\mathrm{C}=\mathrm{N}),{ }^{1} \mathrm{H}$ NMR (DMSO-d $\left.)_{6}\right): \delta=5.80(\mathrm{~s}, 1 \mathrm{H}$, pyridine- $\mathrm{CH}), 6.52-7.88(\mathrm{~m}, 3 \mathrm{H}$, furan- $\mathrm{CH}), 7.00(\mathrm{~s}, 2 \mathrm{H}$, $\mathrm{NH}_{2}, \mathrm{D}_{2} \mathrm{O}$-exchangeable), $7.52(\mathrm{~s}, 1 \mathrm{H}, \mathrm{CH}), 12.56(\mathrm{~s}, 1 \mathrm{H}$, $\mathrm{OH}, \mathrm{D}_{2} \mathrm{O}$-exchangeable). Analysis Calcd for $\mathrm{C}_{11} \mathrm{H}_{8} \mathrm{~N}_{4} \mathrm{O}_{3}$ (244.21): C, 54.10; H, 3.30; N, 22.94\%. Found: C, 54.37; H, 3.42; N, 23.18\%.

Synthesis of hydrazide derivatives 21 and 22.

General procedure: To a mixture of compound $\mathbf{3}$ (0.177 g, $1.0 \mathrm{mmol})$ either acetophenone (19) (0.12 g, 1.0 mmol) or $p$-methoxyacetophenone (20) (0.15 g, 1.0 mmol) a catalytic amount of ammonium acetate $(0.05 \mathrm{~g})$ was added. The reaction mixture was fused at $140^{\circ} \mathrm{C}$ for $15 \mathrm{~min}$, then left to cool. The solid product formed after boiling in dimethylformamide was collected by filtration.

2-Cyano- $N$-(furan-2-ylmethylene)-3-phenylbut-2-en ehydrazide (21). Dark brown crystals from dimethylformamide, $77 \%(0.136 \mathrm{~g})$ yield, $\mathrm{mp} .230^{\circ} \mathrm{C}-232^{\circ} \mathrm{C}$, MS: $\mathrm{m} / \mathrm{z}=279\left(\mathrm{M}^{+}\right)$, IR $(\mathrm{KBr}): \mathrm{v} / \mathrm{cm}^{-1}=3250\left(\mathrm{CH}_{\mathrm{Ar}}\right), 3190$ $(\mathrm{NH}), 3050\left(\mathrm{CH}_{3}\right), 2180(\mathrm{CN}), 1660(\mathrm{CO}), 1560(\mathrm{C}=\mathrm{C})$, ${ }^{1} \mathrm{H}$ NMR $\left(\mathrm{DMSO}_{-} \mathrm{d}_{6}\right): \delta=2.42\left(\mathrm{~s}, 3 \mathrm{H}, \mathrm{CH}_{3}\right), 6.52-7.88$ (m, 3H, furan-CH), $7.33-7.77\left(\mathrm{~m}, 5 \mathrm{H}, \mathrm{H}_{\mathrm{Ar}}\right), 7.00(\mathrm{~s}, 1 \mathrm{H}$, $\mathrm{NH}, \mathrm{D}_{2} \mathrm{O}$-exchangeable), $7.53(\mathrm{~s}, 1 \mathrm{H}, \mathrm{CH})$. Analysis Calcd for $\mathrm{C}_{16} \mathrm{H}_{13} \mathrm{~N}_{3} \mathrm{O}_{2}$ (279.29): C, 68.81; H, 4.69; $\mathrm{N}$, $15.05 \%$. Found: C, 69.73; H, 4.72; N, 14.88\%.

2-Cyano- $N$-(furan-2-ylmethylene)-3-(4-methoxyphenyl) but-2-enehydrazide (22). Dark orange crystals from 1,4-dioxane, $75 \%(0.132 \mathrm{~g})$ yield, $\mathrm{mp} .178^{\circ} \mathrm{C}-180^{\circ} \mathrm{C}$, MS: $\mathrm{m} / \mathrm{z}=309\left(\mathrm{M}^{+}\right)$, IR $(\mathrm{KBr}): \mathrm{v} / \mathrm{cm}^{-1}=3280(\mathrm{NH})$, $3200\left(\mathrm{CH}_{\mathrm{Ar}}\right), 3050,3080\left(2 \mathrm{CH}_{3}\right), 2180(\mathrm{CN}), 1650(\mathrm{CO})$, $1580(\mathrm{C}=\mathrm{C}), 1300,1000(2 \mathrm{C}-\mathrm{O}),{ }^{1} \mathrm{H}$ NMR $\left(\mathrm{DMSO}-\mathrm{d}_{6}\right)$ : $\delta=2.42\left(\mathrm{~s}, 3 \mathrm{H}, \mathrm{CH}_{3}\right), 3.83\left(\mathrm{~s}, 3 \mathrm{H}, \mathrm{CH}_{3}\right), 6.52-7.88(\mathrm{~m}$, $3 \mathrm{H}$, furan-CH), $7.33-7.87\left(\mathrm{~m}, 4 \mathrm{H}, \mathrm{H}_{\mathrm{Ar}}\right), 7.00(\mathrm{~s}, 1 \mathrm{H}, \mathrm{NH}$, $\mathrm{D}_{2} \mathrm{O}$-exchangeable), $7.52(\mathrm{~s}, 1 \mathrm{H}, \mathrm{CH})$. Analysis Calcd for $\mathrm{C}_{17} \mathrm{H}_{15} \mathrm{~N}_{3} \mathrm{O}_{3}$ (309.32): C, 66.01; H, 4.89; N, 15.52\%. Found: C, 65.83; H, 4.63; N, 15.72\%.
Synthesis of phenylhydrazone derivatives 24a-24d.

General procedure: To a cold solution $\left(0^{\circ} \mathrm{C}-5^{\circ} \mathrm{C}\right)$ of compound $3(0.177 \mathrm{~g}, 1.0 \mathrm{mmol})$ in ethanol $(15 \mathrm{~mL})$ an equimolar amount of diazotized benzenediazonium chloride (23a), 4-methylbenzenediazonium chloride (23b), 4chlorobenzenedizonium chloride (23c) and 4-methoxybenzenedizonium chloride (23d) were gradually added under stirring. The solid product separated during stirring in an ice bath was collected by filtration and washed thoroughly with water.

2-[2-(Furan-2-ylmethylene)hydrazinyl]-2-oxo-Ń-ph enylacetohydraz-onoylcyanide (24a). Yellow crystals from 1,4-dioxane, $92 \%(0.162 \mathrm{~g})$ yield, mp. $130^{\circ} \mathrm{C}$ $132^{\circ} \mathrm{C}, \mathrm{MS}: \mathrm{m} / \mathrm{z}=281\left(\mathrm{M}^{+}\right), \operatorname{IR}(\mathrm{KBr}): \mathrm{v} / \mathrm{cm}^{-1}=3280$ $(\mathrm{NH}), 3100\left(\mathrm{CH}_{\mathrm{Ar}}\right), 2160(\mathrm{CN}), 1680(\mathrm{CO}), 1550(\mathrm{C}=\mathrm{N})$, ${ }^{1} \mathrm{H}$ NMR (DMSO-d $\left.{ }_{6}\right): \delta=6.52-8.44\left(\mathrm{~m}, 8 \mathrm{H}, 5 \mathrm{H}_{\mathrm{Ar}}\right.$, furan-3CH), $7.52(\mathrm{~s}, 1 \mathrm{H}, \mathrm{CH}), 10.55,11.61(2 \mathrm{~s}, 2 \mathrm{H}, 2 \mathrm{NH}$, $\mathrm{D}_{2} \mathrm{O}$-exchangeable). Analysis Calcd for $\mathrm{C}_{14} \mathrm{H}_{11} \mathrm{~N}_{5} \mathrm{O}_{2}$ (281.27): C, 59.78; H, 3.94; N, 24.90\%. Found: C, 59.92; H, 4.12; N, 24.69\%.

2-(2-(Furan-2-ylmethylene)hydrazinyl)-2-oxo-Ń-ptolylacetohydraz-onoylcyanide (24b). Pale yellow crystals from 1,4-dioxane, $95 \%(0.168 \mathrm{~g})$ yield, mp. $160^{\circ} \mathrm{C}$ $162^{\circ} \mathrm{C}, \mathrm{MS}: \mathrm{m} / \mathrm{z}=295\left(\mathrm{M}^{+}\right)$, IR $(\mathrm{KBr}): \mathrm{v} / \mathrm{cm}^{-1}=3250$ $(\mathrm{NH}), 3100\left(\mathrm{CH}_{\mathrm{Ar}}\right), 3080\left(\mathrm{CH}_{3}\right), 2150(\mathrm{CN}), 1670(\mathrm{CO})$, $1550(\mathrm{C}=\mathrm{N}),{ }^{1} \mathrm{H}$ NMR $\left(\mathrm{DMSO}_{6}\right): \delta=2.34\left(\mathrm{~s}, 3 \mathrm{H}, \mathrm{CH}_{3}\right)$, $7.53(\mathrm{~s}, 1 \mathrm{H}, \mathrm{CH}), 6.52-8.44\left(\mathrm{~m}, 7 \mathrm{H}, 4 \mathrm{H}_{\mathrm{Ar}}\right.$, furan-3CH), 10.55, $11.61\left(2 \mathrm{~s}, 2 \mathrm{H}, 2 \mathrm{NH}, \mathrm{D}_{2} \mathrm{O}\right.$-exchangeable). Analysis Calcd for $\mathrm{C}_{15} \mathrm{H}_{13} \mathrm{~N}_{5} \mathrm{O}_{2}$ (295.30): C, 61.01; H, 4.44; N, $23.72 \%$. Found: C, 60.82; H, 4.31; N, 23.99\%.

Ń-(4-Chlorophenyl)-2-[2-(furan-2-ylmethylene)hyd razinyl]-2-oxoace-tohydrazonoylcyanide (24c). Orange crystals from 1,4-dioxane, 95\% (0.168 g) yield, mp. $168^{\circ} \mathrm{C}-170^{\circ} \mathrm{C}, \mathrm{MS}: \mathrm{m} / \mathrm{z}=317\left(\mathrm{M}^{+}\right)$, IR $(\mathrm{KBr}): \mathrm{v} / \mathrm{cm}^{-1}=$ $3200(\mathrm{NH}), 3090\left(\mathrm{CH}_{\mathrm{Ar}}\right), 2180(\mathrm{CN}), 1600(\mathrm{CO}), 1550$ $(\mathrm{C}=\mathrm{N}), 1100(\mathrm{C}-\mathrm{Cl}),{ }^{1} \mathrm{H}$ NMR $\left(\mathrm{DMSO}^{-} \mathrm{d}_{6}\right): \delta=7.52(\mathrm{~s}$, $1 \mathrm{H}, \mathrm{CH}=\mathrm{N}), 6.52-8.44\left(\mathrm{~m}, 7 \mathrm{H}, 4 \mathrm{H}_{\mathrm{Ar}}\right.$, furan-3H), 10.54 , $11.62\left(2 \mathrm{~s}, 2 \mathrm{H}, 2 \mathrm{NH}, \mathrm{D}_{2} \mathrm{O}\right.$-exchangeable). Analysis Calcd for $\mathrm{C}_{14} \mathrm{H}_{10} \mathrm{ClN}_{5} \mathrm{O}_{2}$ (315.71): C, 53.26; H, 3.19; N, 22.18\%. Found: C, 53.37; H, 3.28; N, 22.09\%.

2-[2-(Furan-2-ylmethylene)hydrazinyl]-Ń-(4-nitrop henyl)-2-oxoacet-ohydrazonoylcyanide (24d). Reddish brown crystals from 1,4-dioxane, 96\% (0.169 g) yield, mp. $150^{\circ} \mathrm{C}-152^{\circ} \mathrm{C}, \mathrm{MS}: \mathrm{m} / \mathrm{z}=326\left(\mathrm{M}^{+}\right)$, IR $(\mathrm{KBr}): \mathrm{v} / \mathrm{cm}^{-1}$ $=3280(\mathrm{NH}), 3100\left(\mathrm{CH}_{\mathrm{Ar}}\right), 2160(\mathrm{CN}), 1680(\mathrm{CO}), 1550$ $(\mathrm{C}=\mathrm{N}), 1530,1350\left(\mathrm{NO}_{2}\right),{ }^{1} \mathrm{H}$ NMR $\left(\mathrm{DMSO}-\mathrm{d}_{6}\right): \delta=$ $7.52(\mathrm{~s}, 1 \mathrm{H}, \mathrm{CH}), 6.52-8.44\left(\mathrm{~m}, 7 \mathrm{H}, 4 \mathrm{H}_{\mathrm{Ar}}\right.$, furan-3CH), 10.55, $11.61\left(2 \mathrm{~s}, 2 \mathrm{H}, 2 \mathrm{NH}, \mathrm{D}_{2} \mathrm{O}\right.$-exchangeable). Analysis Calcd for $\mathrm{C}_{14} \mathrm{H}_{10} \mathrm{~N}_{6} \mathrm{O}_{4}$ (326.27): C, 51.54; H, 3.09; N, $25.76 \%$. Found: C, 51.67; H, 3.38; N, 25.83\%.

4-Amino-5-cyano- $N$-(furan-2-ylmethylene)-6-oxo-1phenyl-1,6-dihyd-ropyridazine-3-carbohydrazide (26). To a solution of compound $24 \mathrm{a}(0.281 \mathrm{~g}, 1.0 \mathrm{mmol})$ ethyl cyanoacetate $\mathbf{( 8 b )}(0.11 \mathrm{~g}, 1.0 \mathrm{mmol})$ was added in 1,4- 
dioxane $(25 \mathrm{~mL})$ containing triethylamine (4 drops) the reaction mixture was heated under reflux for $4 \mathrm{~h}$, then poured on an ice/water mixture containing a few drops of hydrochloric acid. The solid product formed was collected by filtration.

Dark brown crystals from dimethylformamide, 78\% $(0.219 \mathrm{~g})$ yield, $\mathrm{mp} .250^{\circ} \mathrm{C}-252^{\circ} \mathrm{C}, \mathrm{MS}: \mathrm{m} / \mathrm{z}=348\left(\mathrm{M}^{+}\right)$, IR (KBr): v/cm ${ }^{-1}=3320\left(\mathrm{NH}_{2}\right), 3180(\mathrm{NH}), 2220(\mathrm{CN})$, 1700, $1650(2 \mathrm{CO}), 1550(\mathrm{C}=\mathrm{N}),{ }^{1} \mathrm{H}$ NMR $\left(\mathrm{DMSO}-\mathrm{d}_{6}\right): \delta$ $=6.52-8.48\left(\mathrm{~m}, 8 \mathrm{H}, 5 \mathrm{H}_{\mathrm{Ar}}\right.$, furan-3CH$), 7.10(\mathrm{~s}, 2 \mathrm{H}$, $\left.\mathrm{NH}_{2}\right), 7.50(\mathrm{~s}, 1 \mathrm{H}, \mathrm{CH}), 11.62\left(\mathrm{~s}, 1 \mathrm{H}, \mathrm{NH}, \mathrm{D}_{2} \mathrm{O}-\mathrm{ex}-\right.$ changeable). Analysis Calcd for $\mathrm{C}_{17} \mathrm{H}_{12} \mathrm{~N}_{6} \mathrm{O}_{3}$ (348.32): $\mathrm{C}$, $58.62 ; \mathrm{H}, 3.47$; N, 24.13\%. Found: C, 58.52; H, 3.62; N, $24.40 \%$.

5-[(Furan-2-ylmethylene)hydrazono]-2,4-diphenyl3-thioxo-2,3,4,5-tetrahydro-1,2,4-triazine-6-carbonitrile (29). To a solution of compound $24 \mathrm{a}(0.281 \mathrm{~g}, 1.0 \mathrm{mmol})$ in 1,4-dioxane $(25 \mathrm{~mL})$ containing triethylamine (4 drops) phenyl isothiocynate (27) $(0.13 \mathrm{~g}, 1.0 \mathrm{mmol})$ was added. The reaction mixture was heated under reflux for $3 \mathrm{~h}$, and then poured on an ice/water mixture containing a few drops of hydrochloric acid. The solid product formed was collected by filtration. Dark orange crystals from ethanol, $63 \%(0.177 \mathrm{~g})$ yield, mp. $60^{\circ} \mathrm{C}-62^{\circ} \mathrm{C}$, IR $(\mathrm{KBr}): \mathrm{v} / \mathrm{cm}^{-1}$ $=3050\left(\mathrm{CH}_{\mathrm{Ar}}\right), 2250(\mathrm{CN}), 2150(\mathrm{C}=\mathrm{S}), 1550(\mathrm{C}=\mathrm{N}),{ }^{1} \mathrm{H}$ NMR (DMSO- $\left.\mathrm{d}_{6}\right): \delta=6.25-8.50\left(\mathrm{~m}, 10 \mathrm{H}, 10 \mathrm{H}_{\mathrm{Ar}}\right), 6.52$ - $7.88(\mathrm{~m}, 3 \mathrm{H}$, furan-3CH), $7.51(\mathrm{~s}, 1 \mathrm{H}, \mathrm{CH})$. Analysis Calcd for $\mathrm{C}_{21} \mathrm{H}_{14} \mathrm{~N}_{6} \mathrm{OS}$ (398.44): C, 63.30; H, 3.54; N, $21.09 \%$. Found: C, 63.51; H, 3.22; N, 21.27\%.

Synthesis of thiazole derivatives 33 and 35a,b.

General procedure: To a solution of compound 3 $(0.177 \mathrm{~g}, 1.0 \mathrm{mmol})$ in dimethylformamide $(20 \mathrm{~mL})$ containing potassium hydroxide $(0.05 \mathrm{~g}, 1.0 \mathrm{mmol})$, phenyl isothiocynate (27) $(0.13 \mathrm{~g}, 1.0 \mathrm{mmol})$ was added. The reaction mixture was stirred over night, either ethyl chloroactate (31) $(0.12 \mathrm{~g}, 1.0 \mathrm{mmol})$ or chloroacetone (34a) $(0.09 \mathrm{~g}, 1.0 \mathrm{mmol})$ or phenacyl bromide (34b) $(0.19 \mathrm{~g}, 1.0 \mathrm{mmol})$ was added with continuation of stirring over night. Then the reaction mixture in each case was poured on an ice/water mixture containing a few drops of hydrochloric acid, and then was left over night. The solid product formed in each case was collected by filtration.

2-Cyano- $N$-(furan-2-ylmethylene)-2-(4-hydroxy-3-p henylthiazol-2(3H)-ylidene)acetohydrazide (33). Reddish brown crystals from ethanol/water, $67 \%(0.118 \mathrm{~g})$ yield, $\mathrm{mp} .67^{\circ} \mathrm{C}-69^{\circ} \mathrm{C}$, MS: $\mathrm{m} / \mathrm{z}=352\left(\mathrm{M}^{+}\right)$, IR $(\mathrm{KBr})$ : $\mathrm{v} / \mathrm{cm}^{-1}=3390(\mathrm{OH}), 3280(\mathrm{NH}), 3150\left(\mathrm{CH}_{\mathrm{Ar}}\right), 2150$ $(\mathrm{CN}), 1680(\mathrm{CO}), 1638(\mathrm{C}=\mathrm{C}), 1580(\mathrm{C}=\mathrm{N}),{ }^{1} \mathrm{H}$ NMR $\left(\mathrm{DMSO}^{\mathrm{d}} \mathrm{d}_{6}\right): \delta=5.79(\mathrm{~s}, 1 \mathrm{H}$, thiazole-CH), $6.25-7.20(\mathrm{~m}$, $\left.5 \mathrm{H}, \mathrm{C}_{6} \mathrm{H}_{5}\right), 6.52-7.88(\mathrm{~m}, 3 \mathrm{H}$, furan-3CH), $7.51(\mathrm{~s}, 1 \mathrm{H}$, $\mathrm{CH}), 11.62\left(\mathrm{~s}, 1 \mathrm{H}, \mathrm{NH}, \mathrm{D}_{2} \mathrm{O}\right.$-exchangeable), $12.55(\mathrm{~s}, 1 \mathrm{H}$, $\mathrm{OH}, \mathrm{D}_{2} \mathrm{O}$-exchangeable). Analysis Calcd for $\mathrm{C}_{17} \mathrm{H}_{12} \mathrm{~N}_{4} \mathrm{O}_{3} \mathrm{~S}$ (352.37): C, 57.95; H, 3.43; N, 15.90; S, 9.10\%. Found:
C, 58.28; H, 3.59; N, 16.04; S, 9.39\%.

2-Cyano- $N$-(furan-2-ylmethylene)-2-(4-methyl-3-ph enylthiazol-2(3H)-ylidene)acetohydrazide (35a). Dark brown crystals from acetic acid, 63\% (0.111 g) yield, mp. $80^{\circ} \mathrm{C}-82^{\circ} \mathrm{C}, \mathrm{MS}: \mathrm{m} / \mathrm{z}=350\left(\mathrm{M}^{+}\right), \mathrm{IR}(\mathrm{KBr}): \mathrm{v} / \mathrm{cm}^{-1}=$ $3220(\mathrm{NH}), 2980\left(\mathrm{CH}_{3}\right), 2200(\mathrm{CN}), 1670(\mathrm{CO}), 1640$ $(\mathrm{C}=\mathrm{C}), 1600(\mathrm{C}=\mathrm{N}),{ }^{1} \mathrm{H}$ NMR $\left(\mathrm{DMSO}-\mathrm{d}_{6}\right): \delta=2.35(\mathrm{~s}$, $\left.3 \mathrm{H}, \mathrm{CH}_{3}\right), 5.81(\mathrm{~s}, 1 \mathrm{H}$, thiazole-CH), $6.25-7.20(\mathrm{~m}, 5 \mathrm{H}$, $\left.\mathrm{H}_{\mathrm{Ar}}\right), 6.52-7.88(\mathrm{~m}, 3 \mathrm{H}$, furan-3CH), $7.52(\mathrm{~s}, 1 \mathrm{H}, \mathrm{CH})$ $11.62\left(\mathrm{~s}, 1 \mathrm{H}, \mathrm{NH}, \mathrm{D}_{2} \mathrm{O}\right.$-exchangeable). Analysis Calcd for $\mathrm{C}_{18} \mathrm{H}_{14} \mathrm{~N}_{4} \mathrm{O}_{2} \mathrm{~S}$ (350.39): C, 61.70; H, 4.03; N, 15.99; $\mathrm{S}$, 9.15\%. Found: C, 61.95; H, 3.87; N, 16.21; S, 8.88\%.

2-Cyano-2-(3,4-diphenylthiazol-2(3H)-ylidene)- $N$ (furan-2-ylme-thylene)acetohydrazide (35b). Dark green crystals from acetic acid, $60 \%(0.106 \mathrm{~g})$ yield, $\mathrm{mp} .70^{\circ} \mathrm{C}$ - $72^{\circ} \mathrm{C}$, MS: $\mathrm{m} / \mathrm{z}=412\left(\mathrm{M}^{+}\right), \mathrm{IR}(\mathrm{KBr}): \mathrm{v} / \mathrm{cm}^{-1}=3220$ $(\mathrm{NH}), 3150\left(\mathrm{CH}_{\mathrm{Ar}}\right), 2190(\mathrm{CN}), 1690(\mathrm{CO}), 1600(\mathrm{C}=\mathrm{C})$, $1620(\mathrm{C}=\mathrm{N}),{ }^{1} \mathrm{H}$ NMR $\left(\mathrm{DMSO}_{6}\right): \delta=5.81(\mathrm{~s}, 1 \mathrm{H}$, thiazole $\mathrm{CH}), 6.25-7.20\left(\mathrm{~m}, 5 \mathrm{H}, \mathrm{H}_{\mathrm{Ar}}\right), 6.52-7.88(\mathrm{~m}$, $3 \mathrm{H}$, furan-3CH), $7.52(\mathrm{~s}, 1 \mathrm{H}, \mathrm{CH}), 7.71-7.69(\mathrm{~m}, 5 \mathrm{H}$, $\left.\mathrm{H}_{\mathrm{Ar}}\right), 11.62\left(\mathrm{~s}, 1 \mathrm{H}, \mathrm{NH}, \mathrm{D}_{2} \mathrm{O}\right.$-exchangeable). Analysis Calcd for $\mathrm{C}_{23} \mathrm{H}_{16} \mathrm{~N}_{4} \mathrm{O}_{2} \mathrm{~S}$ (412.46): C, 66.97; H, 43.91; N, 13.58; S, 7.77\%. Found: C, 67.29; H, 3.66; N, 13.72; S, $8.01 \%$.

\section{Acknowledgements}

R. M. Mohareb would like to express his deepest thanks to the Alexander von HumboldtFoundation in Bonn for affording him a fellowship in Germany, LMU Munchen, during summer 2012 for doing research and completling this work.

\section{REFERENCES}

[1] S. Rollas, N. Kalyoncuoğlu, D. Sur-Altiner and Y. Yeğenoğlu, "5-(4-Aminophenyl)-4-substituted-2,4-dihydro-3H1,2,4-triazole-3-thiones: Synthesis and Antibacterial and Antifungal Activities," Pharmazie, Vol. 48, No. 4, 1993, pp. 308-309. doi:10.1016/S0223-5234(99)80048-8

[2] S. Papakonstantinou-Garoufalias, N. Pouli, P.-Marakos and A. Chytyro-Glouladas, "Synthesis Antimicrobial and Antifungal Activity of Some New 3-Substituted Derivatives of 4-(2,4-Dichlorophenyl)-5-adamantyl)-1H-1,2,4-triazole," Il Farmaco, Vol. 57, No. 12, 2002, pp. 973-977. doi:10.1002/chin.200314128

[3] M. Kidwai, R. Kumar, A. Srivastava and H. P. Gupta, "Microwave-Assisted Synthesis of Novel 1,3,4-Thiadiazolyl-Substituted 1,2,4-Triazines as Potential Antitubercular Agents," Bioorganic Chemistry, Vol. 26, No. 5, 1998, pp. 289-294. doi:10.1006/bioo.1998.1108

[4] V. V. Kachhadia, M. R. Patel and H. S. Joshi, "Heterocyclic Systems Containing S/N Regioselective Nucleophilic Competition: Facile Synthesis, Antitubercular and Antimicrobial Activity of THIOHydantoins and Iminothiazolidinones Containing the Benzo[b]thiophene Moiety," Journal of the Serbian Chemical Society, Vol. 70, 
No. 2, 2005, pp. 153-161. doi:10.1002/ardp.201000010

[5] P. K. Kadaba, "Rational Drug Design and the Discovery of the Delta2-1,2,3-triazolines, a Unique Class of Anticonvulsant and Antiischemic Agents," Current Medicinal Chemistry, Vol. 10, No. 20, 2003, pp. 2081-2108. doi:10.2174/0929867033456765

[6] İ. küçükgüzel, Ş. G. Küçükgüzel, S. Rollas, G. Ö.-Saniş, O. Özdemir, I. Bayrak, T. Altuğ and J. P. Stables, "Synthesis of Some 3-(Arylalkylthio)-4-alkyl/aryl-5-(4-aminophenyl)-4H-1,2,4-triazole Derivatives and Their Anticonvulsant Activity," Il Farmaco, Vol. 59, No. 11, 2004, pp. 893-901. doi:10.4103/0975-7406.80783

[7] M. D. Mullican, M. W. Wilson, D. T. Connor, C. R. Kostlan, D. J. Schrier and R. D. Dyer, "Design of 5-(3,5Di-tert-butyl-4-hydroxyl-phenyl)-1,3,4-thiadiazoles, -1,3,4Oxadiazoles, and -1,2,4-Triazoles as Orally-Active, Nonulcerogenic Antiinflammatory Agents," Journal of Medicinal Chemistry, Vol. 36, No. 8, 1993, pp. 1090-1099. doi:10.1021/jm00060a017

[8] E. Palaska, G. Şahin, P. Kelicen, N. T. Durlu and G. Altinok, "Synthesis and Anti-Inflammatory Activity of 1Acylthiosemicarb-azides, 1,3,4-Oxadiazoles, 1,3,4-Thiadiazoles and 1,2,4-Triazole-3-thiones," Il Farmaco, Vol. 57, No. 2, 2002, pp. 101-107. doi:10.1016/S0014-827X(01)01176-4

[9] A.Varvaresou, T. Siatra-Papastaikoudi, A. Tsotinis, A. TsantiliKakoulidou and A. Vamvakides, "Synthesis, Lipophilicity and Biological Evaluation of Indole-Containing Derivatives of 1,3,4-Thiadiazole and 1,2,4-Triazole," Il Farmaco, Vol. 53, No. 5, 1989, pp. 320-326. doi:10.1016/S0014-827X(98)00024-X

[10] B. S. Holla, B. Veerendra, M. K. Shivanada and B. Poojary, "Synthesis Characterization and Anticancer Activity Studies on Some Mannich Bases Derived from 1,2,4Triazoles," European Journal of Medicinal Chemistry, Vol. 38, No. 7-8, 2003, pp. 759-767. doi:10.1016/S0223-5234(03)00128-4

[11] J. H. Lange, H. H. Stuivenberg, H. K. Coolen, T. J. Adolfs, A. C. McCreary, H. G. Keizer, H. C. Wals, W.Veerman, A. J. Borst, W. de Looff, P. C. Verveer and
C. G. Kruse, "Bioisosteric Replacements of the Pyrazole Moiety of Rimonabant: Synthesis, Biological Properties, and Molecular Modeling Investigations of Thiazoles, Triazoles, and Imidazoles as Potent and Selective CB1 Cannabinoid Receptor Antagonists," Journal of Medicinal Chemistry, Vol. 48, No. 6, 2005, pp. 1823-1838. doi:10.1021/jm040843r

[12] R. M. Mohareb, H. Z. Shams and S. I. Aziz, "Novel Synthesis of 4-(Coumarin-3-yl)-1,3-thiazole, 2-(Coumarin-3carbonyl)thieno(2,3-b)pyridine and 2-(Coumarin-3-carbonyl)thiophene Derivatives," Sulfur Letters, Vol. 13, No. 3, 1991, pp. 101-110. doi:10.1002/chin.199238159

[13] R. M. Mohareb, H. Z. Shams and Y. M. Elkholy, "The Use of 4-Phenyl-3-thiosemicarbazide in Heterocyclic Synthesis: Novel Synthesis of Thiazole, Pyrazole and 1,3,4Thiadiazine Derivatives," Phosphorus, Sulfur \& Silicon, Vol. 72, No. 4, 1992, pp. 93-102. doi:10.1002/chin.199319192

[14] H. Z. Shams, Y. M. Elkholy, N. S. Ibrahim and M. H. Elnagdi, "Nitriles in Organic Synthesis: New Routes for Synthesis of Pyridines and Azinothiopyrans," Journal für Praktische Chemie, Vol. 330, No. 5, 1988, pp. 817-819. doi:10.1002/prac.19883300521

[15] S. M. Sherif, R. M. Mohareb, H. Z. Shams and H. M. Gaber, "A Convenient Synthesis of Polyfunctionally Substituted

Benzo[b]thiophen-2-yl-pyrimidine,-pyrazole,-iso- xazole and Pyridazine Derivatives," Journal of Chemical Research, Vol. 1995, 1995, pp. 434-435, 2658-2674.

[16] H. Z. Shams, Y. M. Elkholy, R. A. Azzam and R. M. Mohareb, "Synthetic Potentialities of Thiophene Systems in Heterocyclic Synthesis: Anovel Synthesis of Thieno[2,3b]pyridine Derivatives," Phosphorus, Sulfur \& Silicon, Vol. 155, No. 2, 1999, pp. 215-233. doi:10.1080/10426509908044972

[17] W. W. Wardakhan, H. Z. Shams and H. E. Moustafa, "Synthesis of Polyfunctionally Substituted Thiophene, Thieno[2,3-b]pyridine and Thieno[2,3-d]pyrimidine Derivatives," Phosphorus, Sulfur \& Silicon, Vol. 180, No. 8, 2005, pp. 1815-1827. doi:10.1080/104265090889422 